\title{
Non-gravitational perturbations and evolution of the asteroid main belt
}

\author{
David Vokrouhlický ${ }^{1}$, M. Brož ${ }^{1}$, W.F. Bottke ${ }^{2}$, D. Nesvorný ${ }^{2}$ \\ and A. Morbidelli ${ }^{3}$ \\ ${ }^{1}$ Institute of Astronomy, Charles University, Prague, V Holešovičkách 2, 18000 Prague 8, \\ Czech Republic \\ email: vokrouhl@mbox.cesnet.cz,mira@sirrah.troja.mff.cuni.cz \\ ${ }^{2}$ Southwest Research Institute, 1050, Walnut St., Suite 400, Boulder, CO-80302, USA \\ email: bottke@boulder.swri.edu, davidn@boulder.swri.edu \\ ${ }^{3}$ Observatoire de Nice, Dept. Cassiopee, BP 4229, 06304 Nice Cedex 4, France \\ email: morby@obs-nice.fr
}

\begin{abstract}
Gravity is the most important force to affect the motion of bodies in the Solar system. At small sizes, however, additional forces must be taken into account to explain fine details of their translational and rotational motion, as well as parameters of their populations. This is because the strength of the non-gravitational perturbations typically increases as $\simeq 1 / D$ toward small sizes $D$. The principal perturbation acting on macroscopic main-belt bodies (sizes up to several kilometers for timescales of about billion years) is due to the anisotropic thermal re-radiation of the absorbed sunlight. In orbital dynamics this is known as the Yarkovsky effect, while in rotational dynamics the same physical phenomenon is called the YORP (YarkovskyO'Keefe-Radzievskii-Paddack) effect. We review the main observational implications of the Yarkovsky/YORP effects as understood and evidenced today.
\end{abstract}

Keywords. minor planets, asteroids; interplanetary medium; radiation mechanisms: thermal

\section{Introduction}

The motion of celestial bodies has been a domain of discovery, application and testing of the gravity force for a long time. Less than two centuries ago, the motion of comets and dust particles in their tails required, for the first time, to account for the recoil effect of sublimated gas/dust and the solar radiation pressure. Asteroids and their fragments down to microscopic sizes, although inactive, are susceptible to pressure due to the absorbed, scattered and thermally re-processed solar radiation. At very small sizes, effects of solar wind drag and Lorentz force due to the interplanetary magnetic field become important. Here we review evidence that non-gravitational perturbations affect the orbital and rotational dynamics of asteroids in the main belt. We restrict to this topic, leaving the issue of cometary dynamics and, for the most part, interplanetary dust dynamics to reviews by S.R. Chesley and M.C. Wyatt (this volume).

Common to all non-gravitational effects relevant for our discussion is their $\propto 1 / D$ fading for bodies with large diameter $D$. This is because their strength is directly proportional to the amount of photons or solar wind particles intercepted by their surface that scales as $\propto D^{2}$. Since their mass increases as $\propto D^{3}$, the non-gravitational acceleration has dependence inversely proportional to size. With a finite observation accuracy, and a finite possible orbital evolution period (limited by the Solar system age), this implies that a given non-gravitational perturbation is relevant for bodies up to some largest size only. Similarly, the strength of non-gravitational effects fades at small sizes. This 
occurs for various reasons: e.g. at sub-micron sizes solar visible radiation ceases to interact with dust particles and does not impart momentum to them; similarly, thermal conduction through a centimetre-sized meteoroid becomes efficient enough to produce a uniform temperature and the Yarkovsky force diminishes. This lower limit means that efficiency of non-gravitational perturbations is usually restricted to some finite interval of sizes. Poynting-Robertson (PR) drag appears to be important for particles between

$\simeq 1 \mu \mathrm{m}$ and $\simeq 1 \mathrm{~cm}$, while the Yarkovsky effect dominates in the size range between $\simeq 10 \mathrm{~cm}$ till $\simeq 10 \mathrm{~km}$.

Unlike gravitational perturbations, which can be often approximated using a minimumparameter point-like model, non-gravitational effects depend on a number of parameters, e.g. albedo and other physical constants, rotation state, etc. This makes models involving these effects less than optimum constrained. Still, insufficiency of the gravitational-only models to explain a number of observational facts and the right order-of-magnitude arguments make them credible. The models involving non-gravitational effects thus usually fit statistical parameters of a large sample of objects (such as the near-Earth asteroids or the asteroid families). However, the Yarkovsky effect has also been directly detected in the motion of a single object (Vokrouhlický et al. 2000, 2004b,c; Chesley et al. 2003) - in that case it is possible to estimate the unknown physical parameters of the body.

Given our tight page limit, we restrict ourselves to review recent results on the Yarkovsky and YORP effects, with only a brief comment on how radiation forces affect interplanetary dust particles. More detailed reviews on these topics can be found in: (i) Dermott et al. (2001) who discuss the radiative effects, including the PR drag, for asteroid dust particles and (ii) Bottke et al. (2003) who discuss the thermal effects. A textbook which reviews both subjects is Bertotti et al. (2003).

\section{Solar radiation pressure: dust band orbital evolution}

Space-borne infrared tools of modern astronomy have significantly changed our understanding of the zodiacal cloud, primarily showing it is complex and highly structured. Among these coherent and localized features, the most outstanding are the bands discovered by the IRAS spacecraft (Low et al. 1984), which are associated with dust produced in the main asteroid belt (Dermott et al. 1984). Having been able to match the mid-latitude of the high-inclination dust band at $\simeq 9.35^{\circ}$, Nesvorný et al. (2002) convincingly argued these particles were produced by recent asteroid disruption events like Karin and Veritas (Sec. 5). Yet, the age of these events is significantly more than the dynamical transport time of $10-200 \mu \mathrm{m}$ particles from the main belt. This means the currently observed dust population cannot originate from the parent break-up directly. Since the estimated collisional lifetime of interplanetary dust particles with sizes smaller than $\simeq 150 \mu \mathrm{m}$ is longer than the depletion time by the PR effect (e.g. Grün et al. 1985; Dermott et al. 2001), the dust band population should be significantly affected by the PR drag. A testable prediction from this model is a shallow size distribution because small particles are more efficiently removed from the system by PR drag (e.g. Dermott et al. 2001).

Using numerical integrations, Grogan et al. (2001) built a model of such dynamically evolving dust bands and compared the predicted shape and amplitude of their structures in the several IRAS wavebands $\dagger$. The best fitted value of the power-law index of the incremental mass distribution was $q \simeq-1.4$, significantly less than what is expected for the collisionally evolved population of particles $(q \simeq-11 / 6 \simeq-1.8$; e.g. Dohnanyi

$\dagger$ Note that each of the wavebands preferentially samples radiation emitted by particles of different sizes, allowing thus to characterize their size distribution. 
1969; O'Brien \& Greenberg 2003). The difference between the power-law index of the observed population and that of the collisionally evolved, but otherwise dynamically static, population is about -1 for the size distribution $\ddagger$, and this matches very well the model of strong dynamical depletion by PR drag. Less evident, but often cited, observational argument for PR-drag-dominated depletion of the main asteroid belt grain population in the size range $\simeq(1-100) \mu \mathrm{m}$ is the measurement of the LDEF spacecraft (Love \& Brownlee 1993). The observed shallow mass power-law index $(q \simeq-1.2)$ is interpreted similarly to the Grogan et al. (2001) analysis of the dust band particles. Here, however, a certain fraction of LDEF detected particles might be of cometary origin, which makes interpretation of the results less clear.

\section{Yarkovsky and YORP effects: principle and early applications}

Solar radiation heats cosmic bodies in an asymmetric way: the noon side receives sunlight and gets slightly hotter, while the midnight side is cooling. The temperature distribution on the surface is affected by the ability of the material to conduct heat (thermal inertia), by rotation rate and by its ability to re-emit radiation. It almost always keeps some of irradiation asymmetry (with an exception of very small bodies, $\leqslant 10 \mathrm{~cm}$ for plausible materials, which are kept isothermal by efficient internal heat conduction). As the body re-emits thermally, it experiences a recoil force proportional to the linear momentum carried away by thermal photons; assuming the emitted radiation is in a local equilibrium with the surface material, the infinitesimal force applied on the body is proportional to the fourth power of the surface temperature. The resulting force applied on the whole body is non-zero because of the persistent temperature asymmetry. In this way the body is self-accelerated by its own thermal radiation; this perturbation is called the Yarkovsky force (e.g. Bottke et al. 2003). Since asteroids and their fragments typically have an irregular shape, the very same thermal effect results in a net torque; Rubincam (2000) coined an acronym YORP for the first letters of scientists (Yarkovsky-O'KeefeRadzievskii-Paddack) whose work was directed toward recognizing the importance of this phenomenon in the long-term evolution in asteroid and meteoroid rotation.

The finite value of the surface thermal inertia produces a secular drift of the body's semimajor axis. Direct solar radiation pressure on a body of an arbitrary shape cannot produce such an effect, and even if albedo variations are taken into account, the net semimajor axis change is small (e.g. Vokrouhlický \& Milani 2000). Difficulties in estimating the Yarkovsky-induced semimajor axis drift rate for a given body stems from its dependence on material (thermal) properties and rotation states. Asteroid observations in the infrared and lightcurve analyses can constrain these parameters, but not precisely.

The YORP effect produces a steady change in an asteroid obliquity and rotation rate and can thus significantly modify the distribution of these parameters for small asteroids and meteoroids† (e.g. Rubincam 2000; Vokrouhlický \& Čapek 2002). Unlike in the Yarkovsky effect case, surface thermal inertia is not necessary for YORP to operate secularly; moreover, solar radiation pressure produces a similar perturbation (e.g. Rubincam 2000). It has been however found that thermal inertia does affect the net result (e.g. Capek \& Vokrouhlický 2004). In addition to the Yarkovsky effect parameters, the YORP torque depends sensitively on the body's shape.

$\ddagger$ Note the power law index $b$ of the incremental size distribution is related to the power law index $q$ of the incremental mass distribution via $b=3 q+2$.

$\dagger$ Because the Yarkovsky effect depends also on rotation parameters, there is a complicated interplay between both thermal effects. 
Meteoroid transport from the main belt to Earth was the original motivation to bring the Yarkovsky effect into planetary science (e.g. Burns et al. 1979). That was also the topic of primary interest when Afonso et al. (1995), Rubincam $(1995,1998)$ and Farinella et al. (1998) revived the Yarkovsky effect from years of hibernation. These works put the idea into a modern context where part of the transport scenario is due to gravitational resonant effects. Thus Bottke et al. (2000) numerically studied the interaction of the Yarkovsky migrating meteoroid orbits with a grid of weak resonances in the inner part of the main asteroid belt, while Vokrouhlický \& Farinella (2000) developed a semi-analytical model to fit the observed distribution of cosmic ray exposure ages of chondrites by applying Yarkovsky-driven delivery to the resonances.

In what follows, we review recent Yarkovsky and YORP effect results.

\section{Yarkovsky effect: sustaining mechanism for unstable populations}

A population of asteroids residing on planet-crossing orbits, and on Earth-crossing orbits in particular, must be continously resupplied from some vast reservoir of objects. This is because their orbits are dynamically unstable, with their typical fates being they fall into the Sun, are ejected from the Solar system, or they hit one of the terrestrial planets. The nature of this process, and thus the origin of near-Earth asteroids (NEAs), has been debated for years. Progress in NEAs discovery programs along with new computational facilities over the last decade allowed the construction of a model in which majority of NEAs are former escapees from the main asteroid belt (e.g. Bottke et al. 2000, 2002). Two major constrains on the delivery mechanism(s) follow from this work: (i) there are about 220 multi-kilometre asteroids to be resupplied into the planet-crossing orbits every My, a majority of which comes from the main asteroid belt, and (ii) the cumulative size distribution of the de-biased multi-kilometre population of NEAs can be approximated as $N(<H) \propto 10^{\gamma H}$ with $\gamma \simeq 0.35$ (in the absolute magnitude range $15.5<$ $H<18)$. This latter finding should be compared with the estimated size distribution of the main belt population in the same size range that leads to $\gamma \simeq 0.26$ (e.g. Ivezić et al. 2001).

A standard model for NEA origin, with regard the asteroid source, assumed collisional ejecta being thrown into the major orbital resonances acting as the immediate routes to deliver material onto planet-crossing orbits. However, Zappalà et al. (2002) reviewed population of plausible parent bodies of multi-kilometre fragments near the major resonances and found the mechanism short to explain the constraint (i) above. Moreover, the value 0.35 of the $\gamma$-exponent in this model appears arbitrary and unrelated to the similar exponent of the population in the main asteroid belt. On the other hand, Morbidelli \& Vokrouhlický (2003) developed a model where the bulk of the NEAs is delivered from the main asteroid belt to the adjacent resonance zones by the Yarkovsky effect. They noticed the high rate of 220 multi-kilometre asteroids per My is affordable within this model (with about right proportion of the three resonant routes; Bottke et al. 2000, 2002). Additionally, the difference of the $\gamma$ exponents in the NEA and in the main belt populations appears naturally explained by the fundamental $\propto 1 / D$ size dependence of the Yarkovsky effect strength. Guillens et al. (2002) analysed the observed numbered asteroids on low-inclination orbits in the unstable zone surrounding the $3 / 1$ resonance. Indeed they found about $40 \%$ of these asteroids, with sizes up to $\simeq 15 \mathrm{~km}$, have short dynamical lifetime and will fall in the resonance within $100 \mathrm{My}$; these objects are the "next generation" of NEAs. Though their work does not prove these asteroids were placed onto their unstable orbits by the Yarkovsky effect, they believe this possibility is the most plausible scenario. 


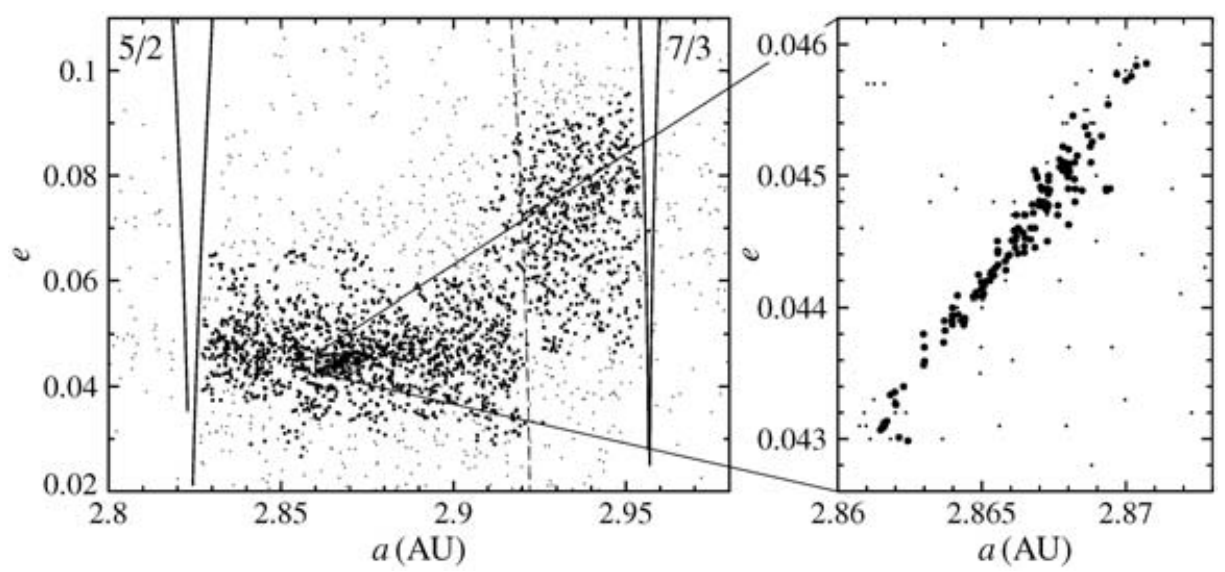

Figure 1. Koronis family and Karin sub-cluster in proper semimajor and eccentricity space (dots are background asteroids and full symbols are the family members). In the left figure, the major mean motion resonances with Jupiter $-5 / 2$ and $7 / 3$ - are shown to bracket the family. Dashed line is a location of the high-order secular resonance $g+2 g_{5}-3 g_{6}$, that makes mean eccentricity in the Prometheus clan (semimajor axes larger than $\simeq 2.92 \mathrm{AU}$ ) higher than for the remaining part of the family. In the right figure we show a zoom on the Karin sub-cluster.

A similar approach has been used to explain the observed dynamically unstable resonant populations via Yarkovsky delivery of small asteroids from the main belt. In particular, Tsiganis et al. (2003) showed a population of objects in the $7 / 3$ mean motion resonance with Jupiter had to be former Koronis and Eos members delivered into their current orbits by the Yarkovsky effect (see below for more details). Brož et al. (2004), using a similar model, succeeded in matching the parameters of asteroids on unstable orbits within the $2 / 1$ mean motion resonance with Jupiter.

\section{Yarkovsky effect: dynamical spreading of asteroid families}

Farinella \& Vokrouhlický (1999) were the first to point out that a large semimajor axis dispersion of small members in the Astrid family might be caused by the spreading of an initially more compact family by the Yarkovsky effect. Though not the best example, this work paved the idea of "Yarkovsky contribution" to the asteroid families evolution. The analysis of the non-gravitational effects in the families is important since that they represent a constrained sample of bodies which were, for the most part, created at the same instant. Though we often do not know when, the common time origin is an important circumstance.

A majority of recent studies have assumed the Yarkovsky effect is the driving mechanism producing the semimajor axis spreading in the families. We note however the work of Dell'Oro et al. (2004) who, by analysing current configuration of families, independently checked their width and determined they must have expanded by a factor of $\simeq 2$ in semimajor axis over time. Here we briefly review the results for three families.

Koronis.- While studying a bizarre shape of this family (Fig. 1), Bottke et al. (2001) brought the first "definitive" proof of Yarkovsky spreading within the asteroid families. First, these authors pointed out that the family identified with a standard clustering methods sharply terminates at the location of $5 / 2$ and $7 / 3$ mean motion resonances with Jupiter. Since these resonances efficiently remove asteroids from the main belt (e.g. Gladman et al. 1997), the shape of the family suggests a permanent flow in the 
semimajor axis from the centre of the family toward the resonances. This is exactly what the Yarkovsky model would predict. However, a major puzzle was the displacement in the mean eccentricity value of Koronis members with $a \geqslant 2.92$ AU (the so called Prometheus clan); no such effect is seen in mean inclination. No reasonable initial velocity field can create such a distorted distribution of fragments. Bottke et al. (2001) proved that the fragments, initially created near the center of the family, migrated outward from the Sun and reached a high-order secular resonance $g+2 g_{5}-3 g_{6}$ that forced their orbital eccentricity $e$ to increase. The amount of this quasi-instantaneous step in $e$ is just right needed to "lift" the asteroids to the Prometheus clan. Since the nodal frequency $s$ does not take part in the resonance frequency, the inclination remains unperturbed.

The Yarkovsky-spreading model implies some fraction of family members should be located at the edge of the major resonances. This was indeed known even earlier, in particular Milani \& Farinella (1995) noticed the asteroid Vysheslavia, a Koronis family member, is located tightly near the $5 / 2$ resonance so that its dynamical lifetime is $\simeq 10 \mathrm{My} \dagger$. This is about two orders of magnitude less than the estimated age of the family. As was concluded by Milani \& Farinella (1995), Vysheslavia is too large $(\simeq 15 \mathrm{~km}$ size) to be a secondary collisional fragment. A more robust scenario is that the body was transported via Yarkovsky effect from stable regions to the 5/2 resonance; Vokrouhlický et al. (2001) determined $\mathrm{a} \sim$ Gy timescale is needed.

Similarly, Tsiganis et al. (2003) recently analysed population of asteroids inside the $7 / 3$ mean motion resonance that brackets the Koronis family (Fig. 1). They noted the population of 23 objects is subdivided in two groups distinct by their orbital inclinations: (i) the low-inclination orbits match the typical values of Koronis members, while (ii) the high-inclination orbits match the typical values of Eos members. With a median dynamical lifetime of several tens of $\mathrm{My}$, this resonant population must be resupplied from the two adjacent families. Importantly, Tsiganis et al. (2003) prove the pace at which the Yarkovsky effect could deliver asteroids from the available Koronis population near the $7 / 3$ resonance is about right to explain the observed low-inclination population (and similarly for the Eos family and the high-inclination resonant population).

Karin.- Nesvorný et al. (2002) noticed an exceptionally compact cluster of asteroids inside the Koronis family (Fig. 1). With a direct integration of their orbits, they proved that the Karin members originated from a single disruptive event $\sim 5.8$ My ago (a similarly spectacular result has been also achieved for the Veritas family; Nesvorný et al. 2003). For the first time, the age of a past disruptive event in the main asteroid belt had been estimated with an uncertainty smaller than few percents. It opened a broad variety of studies. Among them, Nesvorný \& Bottke (2004) dwell on the fact that the proper frequencies $s$ and $g$, with which the proper values of node and perihelion longitudes circulate, depend on the semimajor axis. Since the originally determined convergence of nodes and perihelia $\left(\simeq 40^{\circ}\right.$; Fig. $\left.2 \mathrm{a}\right)$ was nearly an order of magnitude larger than expected just after the disruption event $\neq$, secular drift $d a / d t$ of the semimajor axes may help align them more compactly via additional terms such as $\Delta \Omega_{\text {Yark }} \simeq \frac{1}{2}(\partial s / \partial a)(d a / d t) \tau^{2}$ (Fig. 2b). Here the values of $d a / d t$ appear as a free parameter for each of the asteroids

$\dagger$ Knežević et al. (1997) found two more asteroids with similarly short dynamical lifetime; later Brož \& Vokrouhlický (2001) continued searching in this region and found additional four asteroids.

$\ddagger$ From the semimajor axis, eccentricity and inclination dispersion of Karin members one can easily derive the maximum relative speed of ejection $V_{\mathrm{ej}} \simeq 15 \mathrm{~m} / \mathrm{s}$ (Nesvorný et al. 2002); as an example, Gauss equations then imply the initial node dispersion was $\delta \Omega \simeq 2\left(V_{\mathrm{ej}} / V_{\text {orb }}\right) /(\sqrt{6} \sin I) \simeq 1^{\circ}\left(V_{\text {orb }} \simeq 18 \mathrm{~km} / \mathrm{s}\right.$ is the orbital velocity and $I \simeq 2^{\circ}$ is the inclination). 


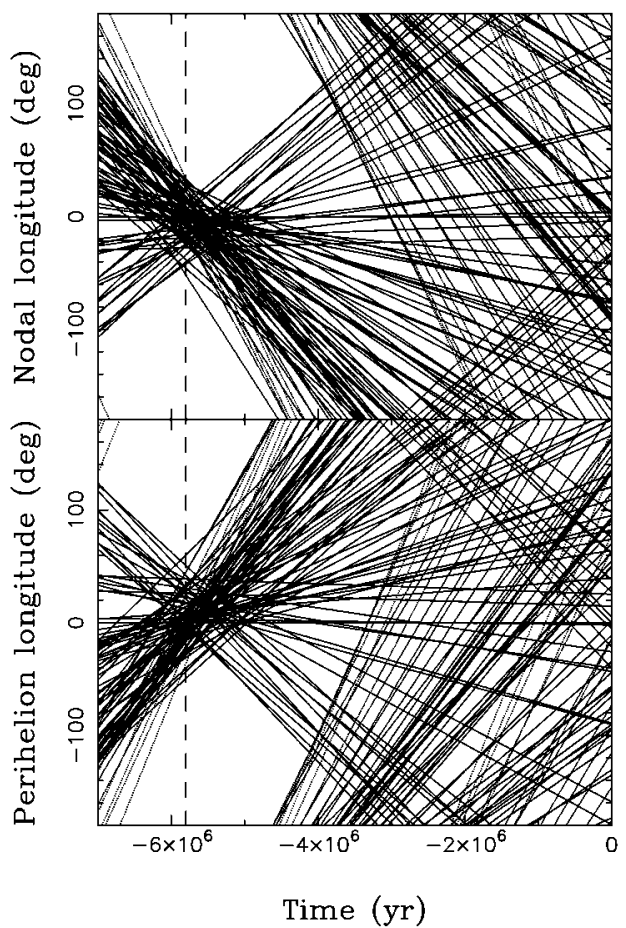

(a)

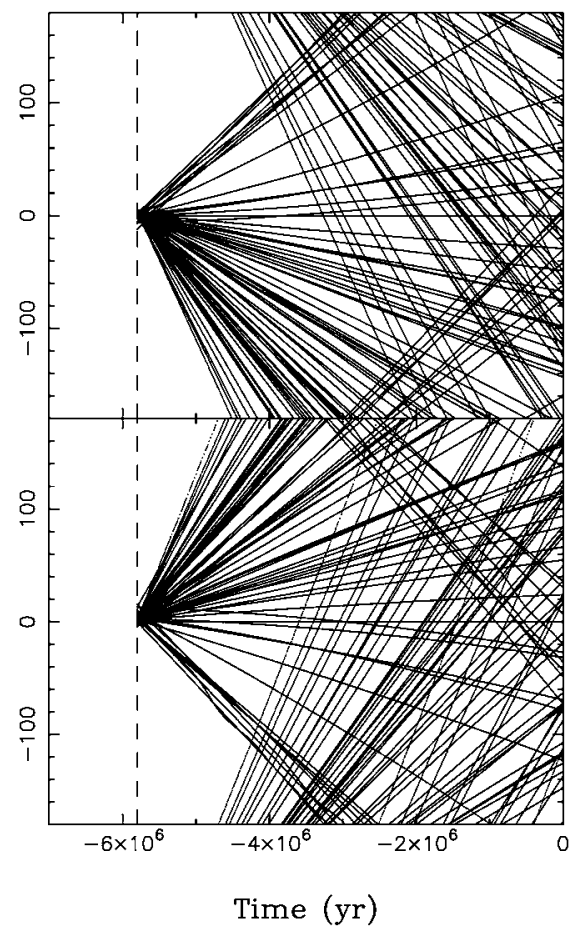

(b)

Figure 2. Convergence of the proper longitude of node and the proper longitude of perihelion for 90 members of Karin cluster at $-5.75 \mathrm{My}$ : (a) without the Yarkovsky effect, (b) with the Yarkovsky effect (Nesvorný \& Bottke 2004). We note a much better convergence of the orbits occurs in the case (b) with a scatter of $\simeq 5^{\circ}$ at the initial time (compared to $\simeq 40^{\circ}$ in the case (a)); a better result is prevented by the gravitational influence of large main belt asteroids whose mass is not known exactly and whose relative motion with respect to the Karin members cannot be modeled deterministically on the My timescale.

making the exercise apparently over-parametrized. However, Nesvorný \& Bottke (2004) noted both proper node and proper perihelion must be aligned for all asteroids and this removes any arbitrariness. Moreover, derived values of $d a / d t$ are of the right order of magnitude expected for the estimated sizes, which further strengthens the case. Extreme values of $d a / d t$ imply the corresponding asteroids must have spin axes nearly perpendicular to their orbits and this is a well-testable prediction. Another important conclusion from this work is that several asteroids whose node and perihelion could not be found convergent with Karin members were proven to be interlopers (among them, the large asteroid (4507) $1990 \mathrm{FV}$ ).

Eos.- Like the Koronis case, the Eos family presented a hard problem for the static family model with its puzzling shape. Morbidelli et al. (1995) pointed out this family is intercepted by the $9 / 4$ mean motion resonance with Jupiter. Numerical integration of fake asteroids inside this resonance indicated their median lifetime of $\simeq 100 \mathrm{My}$ and showed they should be distributed to high eccentricity and inclination values. This finding motivated Zappalà et al. (2000) to search for such asteroids. Spectroscopic similarity with the Eos members should identify them as escaping former family members. Indeed, Zappalà et al. succeeded in discovering 7 positive cases. In fact, this was an initial hint for the ongoing dynamical spreading in this family, since it appears unlikely that the Eos family would be $\simeq 100$ My old. 


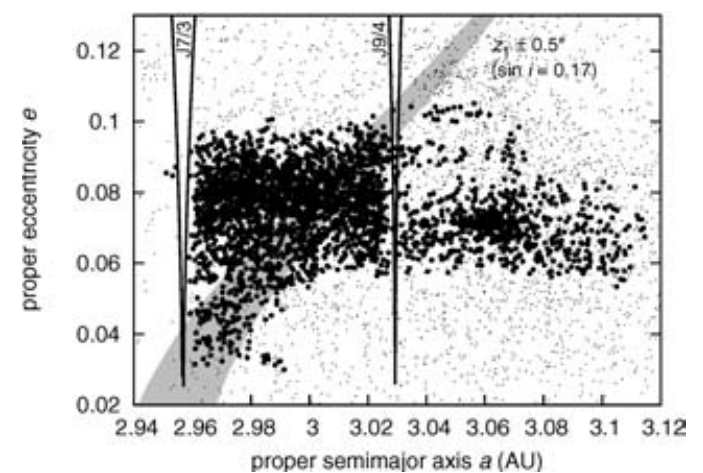

(a)

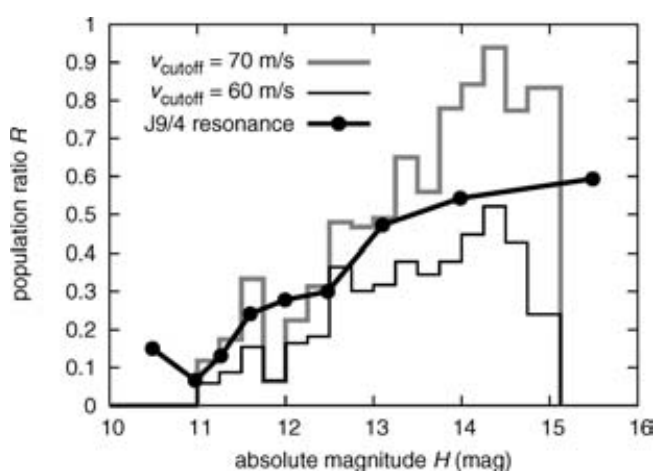

(b)

Figure 3. The Eos family asteroids (full circles) in the projection of proper semimajor axis and eccentricity (left part (a); background asteroids are dots). The shaded zone depicts location of the high-order secular resonance $z_{1}$ for the low inclination edge of the family. On the right part (b) - a relative fraction $R$ of Eos members with higher value of the semimajor axis than the location $9 / 4$ mean motion resonance with respect to the population of asteroids with lower value of the semimajor axis shown as a function of absolute magnitude (the two histograms for two cutoff velocities of the family identification procedure, 60 and $70 \mathrm{~m} / \mathrm{s}$ ). The thick solid curve indicates the numerically determined fraction of Yarkovsky migrating orbits that crossed the resonance.

Further systematic work has been done by Vokrouhlický et al. (2004d; to be submitted) who found four main lines of evidence for Yarkovsky spreading in this family†. First, similar to the Koronis case, the Eos family sharply terminates at the $7 / 3$ mean motion resonance (Fig. 3a). Second, objects with larger semimajor axis value than that of the $9 / 4$ mean motion resonance are under-populated as compared to those with smaller semimajor axis value. This is true even when corrected for the miscentered position of the 9/4 resonance. Vokrouhlický et al. (2004d), however, prove that the observed under-population corresponds to the probability to cross the $9 / 4$ resonance with outward migrating orbits due to the Yarkovsky effect (Fig. 3b). Third, small members in the family are more dispersed in semimajor axis than large members in a way consistent with Yarkovsky evolution. Although one would also assume that the small members acquired initially larger relative velocities, detailed analysis supports a model where the initial semimajor axis dispersion was doubled by the subsequent Yarkovsky migration of small family members. This is because small asteroids tend to populate regions with extreme values of the semimajor axis and under-populate the central zone of the family. Assuming the YORP effect preferentially tilts the obliquity of these objects toward extreme values of $0^{\circ}$ and $180^{\circ}$ (Čapek \& Vokrouhlický 2004), the Yarkovsky drift becomes maximum and produces the observed central depletion. Performing a fit to the observed distribution of the Eos members and using a combined Yarkovsky/YORP model, Vokrouhlický et al. (2004d) determine the age of this family of $(1.3 \pm 0.2)$ Gy. The initial semimajor axis dispersion was found to be about half of the current value which agrees with results in Dell'Oro et al. (2004). Finally, the shape of the Eos family is found to adhere to the high-order secular resonance $z_{1}=s+g-s_{6}-g_{6}$ at small inclinations (Fig. 3a). This appears anomalous in a static model, while it is expected within the Yarkovsky dispersal model because orbits migrating towards smaller values of the semimajor axis are found to be efficiently trapped in this resonance (see also Vokrouhlický \& Brož 2002).

$\dagger$ Preliminary analysis has been reported by us at the ACM 2002 meeting in Berlin, see abstract 12-01, p. 115 of the ACM Abstract Book. 


\section{YORP effect: transport into resonant states}

Compared to the Yarkovsky effect, we currently have less evidence about the YORP effect, in part because its revival came years later (Rubincam 2000). Efforts toward its direct detection via precise observations of the rotation rate of small NEAs have been so far unsuccessfulł. With that, the most convincing evidence about a long-term YORP perturbation comes from an analysis of the spin axis distribution of Koronis family members.

Concluding a long-term observation survey, Slivan (2002) and Slivan et al. (2003) reported astonishing results about the rotation state of several small members of the Koronis family. First, and the most strange, four asteroids with a prograde sense of rotation all have spin axes nearly parallel in space, with obliquity near $55^{\circ}$, and have all rotation periods clustered in a tight range of values between $7.5 \mathrm{hr}$ and $9.5 \mathrm{hr}$. Second, five asteroids with a retrograde sense of rotation have obliquities pushed near the maximum value of $180^{\circ}$ and their rotation periods are anomalously short or long. Since these results are not a random fluke, they call out for a robust theoretical model.

The YORP renaissance came right on time to meet this interesting problem. Vokrouhlický et al. (2003) proved the prograde group of asteroids resides in a secular spin-orbit resonance. This means their spin axes follow a particular mode (here related to the $s_{6}$ frequency) of the mean orbital plane precession in space; in classical astronomy this is the Cassini state 2 (e.g. Colombo 1966). Because this mode is forced by planetary perturbations, it acts the same on all asteroids and this naturally explains the alignment of their spin axes in ecliptic longitude (and thus their true collinearity in space). With that, however, the problem is not solved; we still need to explain why the prograde asteroids are captured by this fine resonance. Here the YORP effect became an instrumental part of the model. Vokrouhlický et al. (2003) proved that in $2-3$ Gy of evolution, a generic initial prograde rotation state (with rotation periods $\simeq 4-9 \mathrm{hr}$ ) evolves toward the $s_{6}$ secular resonance. The YORP torque first tilts the obliquity to a small value, where the capture probability becomes unity; then YORP continues to push evolution toward the stable point of the resonance. The generalized Cassini state, with YORP effect included, has necessarily a resonant obliquity near $55^{\circ} \dagger$; this explains the observed obliquities and also provides evidence that the YORP effect is the underlying dynamical mechanism. The resonant state was found weakly unstable, such that the future evolution will drive the asteroidal spin axes out of its proximity. This has likely already happened to smaller unobserved asteroids, where the strength of the YORP effect is larger. Spin axes of the retrograde group of asteroids evolve toward extreme values in both obliquity and rotation period by YORP since there is no obvious spin-orbit resonance in this zone. Hence, the rotation states of the retrograde asteroids also point to past YORP evolution. Note that this model was further supported by new observations in 2004 (S. Slivan, personal communication).

$\ddagger$ With a near certainty the YORP and the Yarkovsky effects will be detected using Hayabusa observations as a slight deceleration of (25143) Itokawa's rotation and about a $20 \mathrm{~km}$ orbit displacement in the late 2005 (e.g. Vokrouhlický et al. 2004a; Ostro et al. 2004). The analysis of an early photometry of (433) Eros by J. Durech, p. 95 of the Abstracts book, suggests YORP might have been affecting rotation of this asteroid. This empirical result for the Eros rotation period increase compares surprisingly well with the prediction by Čapek \& Vokrouhlický (2004), but large observational errors prevent a clear-cut conclusion.

$\dagger$ In spite of the solid numerical evidence about this result, it has never been theoretically explained why the YORP torque on rotation speed vanishes at this obliquity value. It remains an interesting problem for the future work. 


\section{YORP effect: rotation period and obliquity distribution}

As pointed out above, a fundamental advantage in analyzing asteroid family members is their common age. The YORP effect perturbs the rotation of any small asteroid in the main belt, but their "evolution clocks" are not synchronized to bring them, at a certain time, to a similar evolution state. An interesting exception are NEAs, because most of them recently $(\simeq 1-50$ My ago; Bottke et al. 2002) left the main asteroid belt via three major routes: (i) the $3 / 1$ mean motion resonance with Jupiter, (ii) the $\nu_{6}$ secular resonance, and (iii) the intermediate Mars-crossing zone. Assuming the Yarkovsky effect is the fundamental force that drives asteroids into these escape routes, as suggested by Morbidelli \& Vokrouhlický (2003), one tentatively expects roughly 2/3 of NEAs should preferentially rotate retrograde and $1 / 3$ prograde. This is because with retrograde rotation the Yarkovsky effect causes the asteroids to migrate inwards to the Sun, while prograde rotation makes them to migrate outward from the Sun. Only one of the three options for the transport resonant routes, the 3/1 resonance for inner belt asteroids, is populated by the outward migrating objects. This argument assumes the same number of asteroids leaks through the three escape routes (i) - (iii); when corrected for their relative weight (derived by Bottke et al. 2000, 2002), one would predict $71 \%$ of NEAs have prograde rotation and $29 \%$ of NEAs have retrograde rotation.

This rather rough prediction has been confirmed by the work of La Spina et al. (2004) who find that the ratio of retrograde vs. prograde rotating NEAs is indeed 2:1 with a high statistical confidence. Though more observations are certainly needed to make the current sample of 21 asteroids more sound, this result complements previous lines of evidence for the ongoing Yarkovsky perturbation of orbits of the main belt asteroids.

\section{Conclusions}

The last decade provided ample evidence about ongoing dynamical evolution in the main asteroid belt, with several examples mentioned above. Still, a number of topics remain for further work:

- make use of the Yarkovsky and YORP effects to build a chronology of asteroid families (e.g. Carruba et al. 2003);

- make use of the Yarkovsky and YORP effect to investigate the role of dynamical depletion for analysis of the size distribution function of both the main belt and NEA populations (see W.F. Bottke et al., this volume);

- do the Yarkovsky and YORP effects significantly limit lifetime of (compact) asteroid binaries?

- further detections of the Yarkovsky effect as a systematic tool for asteroid mass determination (e.g. Vokrouhlický et al. 2004b,c);

- searching for more opportunities for a direct detection of the YORP effect (e.g. Vokrouhlický et al. 2004a);

- does YORP produce a significant fraction of slowly tumbling asteroids?

- does YORP produce a significant fraction of compact binaries among the near-Earth asteroids?

The overall importance of these problems for planetary science promise the analysis of the non-gravitational effects will continue to be an active research field over the next decade.

\section{Acknowledgements}

The work of DV and MB was supported by the Grant Agency of the Czech Republic. 


\section{References}

Afonso, G.B., Gomes, R.S. \& Florczak, M.A. 1995, Planet. Sp. Sci. 43, 787

Bertotti, B., Farinella, P. \& Vokrouhlický, D. 2003, Physics of the Solar System (Kluwer)

Bottke, W.F., Rubincam, D.P. \& Burns, J.A. 2000, Icarus 145, 301

Bottke, W.F., Jedicke, R., Morbidelli, A., Petit, J.-M. \& Gladman, B.J. 2000, Science 288, 2190

Bottke, W.F., Vokrouhlický, D., Brož, M., Nesvorný, D. \& Morbidelli, A. 2001, Science 294, 1693

Bottke, W.F., Morbidelli, A., Jedicke, R., Petit, J.-M., Levison, H.F., Michel, P. \& Metcalfe, T.S. 2002, Icarus 156,399

Bottke, W.F., Vokrouhlický, D., Rubincam, D.P. \& Brož, M. 2003, in: Bottke, W.F., Cellino, A., Paolicchi, P. \& Binzel, R.P. (eds.), Asteroids III (The University of Arizona Press, Tucson), p. 395

Brož, M. \& Vokrouhlický, D. 2001, in: Pretka-Ziomek, H., Wnuk, E., Seidelmann, P.K. \& Richardson, D. (eds.), Dynamics of Natural and Artificial Celestial Bodies (Kluwer Academic), p. 307

Brož, M., Vokrouhlický, D., Roig, F., Nesvorný, D., Bottke, W.F. \& Morbidelli, A. 2004, Mon. Not. R. Astron. Soc., submitted

Burns, J.A., Lamy, P.L. \& Soter, S. 1979, Icarus 40, 1

Čapek, D. \& Vokrouhlický, D. 2004, Icarus, in press

Carruba, V., Burns, J.A., Bottke, W.F. \& Nesvorný, D. 2003, Icarus 162, 308

Chesley, S.R., Ostro, S.J., Vokrouhlický, D., Čapek, D., Giorgini, J.D., Nolan, M.C., Margot, J-L., Hine, A.A., Benner, L.A.M. \& Chamberlin, A.B. 2003, Science 302, 1739

Colombo, G. 1966, Astron. J. 71, 891

Dell'Oro, A., Bigongiari, G., Paolicchi, P. \& Cellino, A. 2004, Icarus 169, 341

Dermott, S.F., Nicholson, P.D., Burns, J.A. \& Houck, J.R. 1984, Nature 312, 505

Dermott, S.F., Grogan, K., Durda, D.D., Jayaraman, S., Kehoe, T.J.J., Kortenkamp, S.J. \& Wyatt, M.C. 2001, in: Grün, E., Gustafson, B.A.S., Dermott, S.F. \& Fechtig, H. (eds.), Interplanetary Dust (Springer), p. 569

Dohnanyi, J.W. 1969, J. Geophys. Res. 74, 2531

Farinella, P. \& Vokrouhlický, D. 1999, Science 283, 1507

Farinella, P., Vokrouhlický, D. \& Hartmann, W.K. 1998, Icarus 132, 378

Giorgini, J.D., Ostro, S.J., Benner, L.A.M., Chodas, P.W., Chesley, S.R., Hudson, R.S., Nolan, M.C., Klemola, A.R., Standish, E.M., Jurgens, R.F., Rose, R., Chamberlin, A.B., Yeomans, D.K. \& Margot, J-L. 2002, Science 296, 132

Gladman, B.J., Migliorini, F., Morbidelli, A., Zappalà, V., Michel, P., Cellino, A., Froeschlé, C., Levison, H.F., Bailey, M. \& Duncan, M. 1997, Science 277, 197

Guillens, S.A., Vieira Martins, R. \& Gomes, R.S. 2002, Astron. J. 124, 2322

Grogan, K., Dermott, S.F. \& Durda, D.D. Icarus 152, 251

Grün, E., Zook, H.A., Fechtig, H. \& Giese, R.H. 1985, Icarus 62, 244

Ivezić, Z. and 31 coauthors 2001, Astron. J. 122, 2749

Knežević, Z., Milani, A. \& Farinella, P. 1997, Planet. Sp. Sci. 45, 1581

La Spina, A., Paolicchi, P., Kryszczynska, A. \& Pravec, P. 2004, Nature 428, 400

Love, S.G. \& Brownlee, D.E. 1993, Science 262, 550

Low, F.J., Young, E., Beintema, D.A., Gautier, T.N., Beichman, C.A., Aumann, H.H., Gillett, F.C., Neugebauer, G., Boggess, N. \& Emerson, J. P. 1984, Astrophys. J. Lett. 278, 19L

Morbidelli, A. \& Vokrouhlický, D. 2002, Icarus 163, 120

Morbidelli, A., Zappalà, V., Moons, M., Cellino, A. \& Gonczi, R. 1995, Icarus 118, 132

Nesvorný, D. \& Bottke, W.F. 2004, Icarus 170, 324

Nesvorný, D., Bottke, W.F., Dones, L. \& Levison, H.F. 2002, Nature 417, 720

Nesvorný, D., Bottke, W.F., Levison, H.F. \& Dones, L. 2003, Astrophys. J. 591, 486

O'Brien, D.P. \& Greenberg, R. 2003, Icarus 164, 334

Ostro, S.J., Benner, L.A.M., Nolan, M.C., Magri, C., Giorgini, J.D., Scheeres, D., Broschart, S., Kaasalainen, M., Vokrouhlický, D., Chesley, S.R., Margot, J.-L., Jurgens, R., Rose, A., Yeomans, D., Suzuki, S. \& DeJong, E. 2004, Meteor. Planet. Science 39, 407

Rubincam, D.P. 1995, J. Geophys. Res. 100, 1585 
Rubincam, D.P. 1998, J. Geophys. Res. 103, 1725

Rubincam, D.P. 2000, Icarus 148, 2

Slivan, S.M. 2002, Nature 419, 49

Slivan, S.M., Binzel, R.P., Crespo da Silva, L.D., Kaasalainen, M., Lyndaker, M.M. \& Krčo, M. 2003, Icarus 162, 285

Tsiganis, K., Varvoglis, H. \& Morbidelli, A. 2003, Icarus 166, 131

Vokrouhlický, D. \& Farinella, P. 2000, Nature 407, 606

Vokrouhlický, D. \& Milani, A. 2000, Astron. Astrophys. 362, 746

Vokrouhlický, D. \& Čapek, D. 2002, Icarus 159, 449

Vokrouhlický, D. \& Brož, M. 2002, in: Celletti, A., Ferraz-Mello, S. \& Henrard, J. (eds.), Modern Celestial Mechanics: from Theory to Applications (Kluwer Academic), p. 467

Vokrouhlický, D., Milani, A. \& Chesley, S.R. 2000, Icarus 148, 118

Vokrouhlický, D., Nesvorný, D. \& Bottke, W.F. 2003, Nature 425, 147

Vokrouhlický, D., Brož, M., Farinella, P. \& Knežević, Z. 2001, Icarus 150, 78

Vokrouhlický, D., Čapek, D., Kaasalainen, M. \& Ostro, S.J. 2004a, Astron. Astrophys. 414, L21

Vokrouhlický, D., Čapek, D., Chesley, S.R. \& Ostro, S.J. 2004b, Icarus, in press

Vokrouhlický, D., Čapek, D., Chesley, S.R. \& Ostro, S.J. 2004c, Icarus, submitted

Zappalà, V., Cellino, A. \& Dell'Oro, A. 2002, Icarus 157, 280

Zappalà, V., Bendjoya, P., Cellino, A., Di Martino, M., Doressoundiram, A., Manara, A. \& Migliorini, F. 2000, Icarus 145, 4 